\title{
Donors for SARS-CoV-2 Convalescent Plasma for a Controlled Clinical Trial: Donor Characteristics, Content and Time Course of SARS-CoV-2 Neutralizing Antibodies
}

\author{
Sixten Körper ${ }^{\mathrm{a}, \mathrm{b}}$ Bernd Jahrsdörfer ${ }^{\mathrm{a}, \mathrm{b}}$ Victor M. Corman ${ }^{\mathrm{c}}$ Jan Pilch ${ }^{\mathrm{d}}$ Patrick Wuchter $^{\mathrm{e}}$ \\ Rainer Blasczyk ${ }^{f}$ Rebecca Müller ${ }^{\mathrm{e}}$ Torsten Tonn $^{\mathrm{g}}$ Tamam Bakchoul $^{\text {h }}$ Richard Schäfer $^{\mathrm{i}}$ \\ David Juhl ${ }^{j}$ Tatjana Schwarz ${ }^{c}$ Nina Gödecke ${ }^{f}$ Thomas Burkhardt ${ }^{g}$ Michael Schmidt ${ }^{i}$ \\ Thomas Appl ${ }^{\mathrm{a}, \mathrm{b}} \quad$ Hermann Eichler $^{\mathrm{d}}$ Harald Klüter $^{\mathrm{e}}$ Christian Drosten $^{c}$ Erhard Seifried $^{\mathrm{i}}$ \\ Hubert Schrezenmeier ${ }^{a, b}$ \\ anstitute for Clinical Transfusion Medicine and Immunogenetics Ulm, German Red Cross Blood Transfusion Service \\ Baden-Württemberg-Hessen and University Hospital Ulm, Ulm, Germany; ${ }^{b}$ Institute of Transfusion Medicine, \\ University of Ulm, Ulm, Germany; ${ }^{C}$ Institute of Virology, Charité-Universitätsmedizin Berlin, corporate member \\ of Freie Universität Berlin, Humboldt-Universität zu Berlin, and Berlin Institute of Health and German Centre for

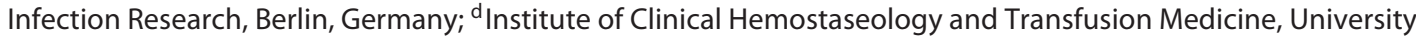

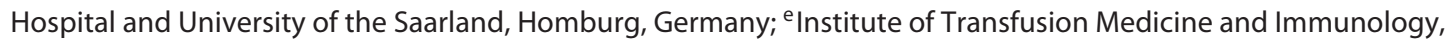 \\ German Red Cross Blood Transfusion Service Baden-Württemberg-Hessen, Medical Faculty of Medicine Mannheim, \\ University Mannheim, Mannheim, Germany; Institute of Transfusion Medicine and Transplant Engineering, \\ Hannover Medical School, Hannover, Germany; ${ }^{9}$ Experimental Transfusion Medicine, Technical University of \\ Dresden, German Red Cross Blood Transfusion Service Nord-Ost gGmbH Dresden, Dresden, Germany; ${ }^{\text {h } I n s t i t u t e}$ \\ of Clinical and Experimental Transfusion Medicine, University Hospital Tübingen, Tübingen, Germany; 'Institute of \\ Transfusion Medicine and Immunohematology, German Red Cross Blood Transfusion Service \\ Baden-Württemberg - Hessen, Frankfurt, Germany; ${ }^{j}$ Institute of Transfusion Medicine, University Hospital \\ Schleswig-Holstein, Kiel and Lübeck, Germany
}

\section{Keywords}

COVID-19 - Convalescent plasma - Plasma donors .

SARS-CoV-2 $\cdot$ Neutralizing antibody

\begin{abstract}
Background: Convalescent plasma is one of the treatment options for COVID-19 which is currently being investigated in many clinical trials. Understanding of donor and product characteristics is important for optimization of convalescent plasma. Methods: Patients who had recovered from COVID-19 were recruited as donors for COVID-19 convalescent plasma (CCP) for a randomized clinical trial of CCP for treatment of severe COVID-19 (CAPSID Trial). Titers of neutralizing antibodies were measured by a plaque-reduction neutralization test (PRNT). Correlation of antibody titers with
\end{abstract}

host factors and evolution of neutralizing antibody titers over time in repeat donors were analysed. Results: A series of 144 donors ( $41 \%$ females, 59\% males; median age 40 years) underwent 319 plasmapheresis procedures providing a median collection volume of $850 \mathrm{~mL}$ and a mean number of 2.7 therapeutic units per plasmapheresis. The majority of donors had a mild or moderate course of COVID-19. The titers of neutralizing antibodies varied greatly between CCP donors (from $<1: 20$ to $>1: 640$ ). Donor factors (gender, age, $A B O$ type, body weight) did not correlate significantly with the titer of neutralizing antibodies. We observed a significant positive correlation of neutralization titers with the number of reported COVID-19 symptoms and with the time

S.K., B.J., and V.M.C. contributed equally to this work.
Correspondence to:

Hubert Schrezenmeier, h.schrezenmeier@blutspende.de 
from SARS-CoV-2 diagnosis to plasmapheresis. Neutralizing antibody levels were stable or increased over time in $58 \%$ of repeat CCP donors. Mean titers of neutralizing antibodies of first donation and last donation of repeat CCP donors did not differ significantly (1:86 at first compared to 1:87 at the last donation). There was a significant correlation of neutralizing antibodies measured by PRNT and anti-SARS-CoV-2 IgG and $\lg A$ antibodies which were measured by ELISA. CCP donations with an anti-SARS-CoV-2 IgG antibody content above the 25th percentile were substantially enriched for CCP donations with higher neutralizing antibody levels. Conclusion: We demonstrate the feasibility of collection of a large number of CCP products under a harmonized protocol for a randomized clinical trial. Titers of neutralizing antibodies were stable or increased over time in a subgroup of repeat donors. A history of higher number of COVID-19 symptoms and higher levels of anti-SARS-CoV-2 IgG and IgA antibodies in immunoassays can preselect donations with higher neutralizing capacity.

(c) 2021 The Author(s)

Published by S. Karger AG, Basel

\section{Introduction}

In spring 2020, we initiated a randomized, prospective, open label clinical trial on the use of convalescent plasma compared to best supportive care in patients with severe COVID-19 (CAPSID; Eudra-CT 2020-001380-00, NCT04433910). Collection of COVID-19 convalescent plasma (CCP) is part of the clinical trial protocol. Here, we report the data on CCP donors and CCP products which have been collected under a stringent clinical trial protocol.

Currently, it is too early to assess the safety and efficacy of CCP for prophylaxis or treatment of COVID-19. Some non-randomized trials [1], a large case series from the US Early Access Programme (EAP) [2], propensity score matching study [3] and some randomized clinical trials have been published [1,4-9]. The data so far are inconsistent. While some of these studies report favourable results at least for some endpoints or subgroups in posthoc analyses $[4,6,10]$, others failed to meet the endpoints $[5,7,8]$. It is important to note that 4 out of 6 randomized trials published so far have been terminated early for various reasons, for example, slow accrual $[4-6,9]$. Several reasons can explain the heterogeneous and contradictory results: differences in the patient populations regarding severity of COVID-19 or the timing of CCP administration during the clinical course of SARS-CoV-2 infection, sample size aspects, but also the dose and quality of the investigational drug CCP $[4-9,11]$.

Therefore, it is important not only to focus on the clinical endpoints of a CCP trial, but also to describe the do- nor population, general CCP product characteristics and in particular the antibody content of CCP. We studied the impact of donor characteristic (age, gender, blood group, severity of COVID-19, interval between SARS-CoV-2 diagnosis and CCP collection) on the antibody content in the CCP products. Donor criteria which predict the antibody titer in CCP could be very helpful to set up an efficient CCP donor accrual programme in the specific context of limited resources during a pandemic. Many CCP donors are motivated to donate several times. Thus, not only the baseline characteristics of the donors but the evolution of CCP characteristics over consecutive plasmapheresis procedures needs to be studied.

We analysed these aspects in a cohort of 144 CCP donors who donated at different donation centres according to the CAPSID trial protocol. Our experience demonstrates the feasibility of collection of a large number of CCP units for a randomized clinical trial during a pandemic. Various demographic donor characteristics were correlated with antibody titers. Most importantly, we observed stable levels of IgG and IgA antibody or neutralizing antibodies over time in a substantial proportion of repeat plasmapheresis donors. We demonstrate feasibility of collection of large number of CCP products under a harmonized protocol.

\section{Donors and Methods}

\section{Donors}

CCP donors were recruited within the clinical trial "A randomized, prospective, open label clinical trial on the use of convalescent plasma compared to best supportive care in patients with severe COVID-19"(CAPSID;Eudra-CT2020-001310-38,NCT04433910). The trial design has been published elsewhere [12]. Plasma donations and blood samples were collected after informed consent.

The detailed criteria for acceptance of a recovered patient to be included as a CCP donor were as follows:

- Infection with SARS-CoV-2 documented by a positive RTPCR (from nasal or pharyngeal swap, bronchoalveolar lavage or stool)

- Proven to have cleared SARS-CoV-2 from nasopharyngeal mucosa by one negative RT-PCR result from a nasal or pharyngeal swab prior to start of first plasmapheresis

- Interval of at least 2 weeks since resolution of symptoms of the SARS-CoV-2 infection

- No residual severe organ dysfunction

- Written informed consent to donate plasma for the clinical trial

- Negative test for antibodies against HLA class I, class II and HNA-antigens. This test was performed in all donors - irrespective of gender and previous pregnancies. It was performed prior to first plasmapheresis and repeated after an immunization event.

- Anti-SARS-CoV-2 antibodies detectable in a neutralization assay. This criterion was dispensable for the first plasmapheresis and respective tests were performed on a sample drawn prior to first plasmapheresis. A donor should continue plasma donation only in case that presence of anti-SARS-CoV-2 antibodies 
is confirmed on pre-plasmapheresis samples prior to first plasmapheresis.

In addition to these criteria, study-specific eligibility criteria, which are the standard criteria for approval as a plasma donor according to the national German Guidelines ("Richtlinie Hämotherapie") [13], had to be observed at each donation:

- Age: first donors: 18-60 years, repeated donors: 18-68 years

- Body weight: $\geq 50 \mathrm{~kg}$

- Overall impression: no obvious signs of disease

- Haemoglobin concentration: women: $\geq 125 \mathrm{~g} / \mathrm{L}$, men: $\geq 135 \mathrm{~g} / \mathrm{L}$

- Leukocytes, erythrocytes, platelets, mean corpuscular volume: within normal range

- Total serum protein (prior to first plasmapheresis and on occasion of every fifth donation): $\geq 60 \mathrm{~g} / \mathrm{L}$

- IgG concentration in serum (prior to first plasmapheresis and on occasion of every fifth donation): $\geq 6.0 \mathrm{~g} / \mathrm{L}$. In case total IgG levels are below $6.0 \mathrm{~g} / \mathrm{L}$ the interval until the next plasmapheresis has to be extended by at least 2 weeks

- Blood pressure: systolic: $100-180 \mathrm{~mm} \mathrm{Hg}$, diastolic: < 100 $\mathrm{mm} \mathrm{Hg}$

- Heart rate: rhythmic, rate $50-110 / \mathrm{min}$; a heart rate below $50 /$ min might be accepted when donors do a lot of sports

- No fever

- Skin (at side of venipuncture): no lesion.

Infectious disease markers were tested for all donations (single sample PCR-testing for hepatitis A, B, C, HIV [14, 15]; PCR for HEV and Parvovirus B19; anti-HCV, anti-HIV, HBsAg, TPHA). A RT-PCR for SARS-CoV-2 was performed from a blood sample drawn prior to the first donation. No pathogen-inactivation was applied.

Also the procedure for plasmapheresis, frequency and intervals of plasmapheresis procedures, was performed according to the "Richtlinie Hämotherapie" [13].

The volume of plasma donation was adapted to the body weight (bw) of donors: $650 \mathrm{~mL}$ in donors with bw from 50 to $60 \mathrm{~kg}, 750$ $\mathrm{mL}$ in donors with bw $>60-80 \mathrm{~kg} ; 850 \mathrm{~mL}$ in donors with bw $>80$ $\mathrm{kg}$. The collection volume was divided into therapeutic units with a target volume of $250-325 \mathrm{~mL}$ plasma per unit.

Plaque Reduction Neutralisation Test for SARS-CoV-2

Plaque reduction neutralisation tests (PRNT) for SARS-CoV-2 were performed as previously described before [16-18]. Briefly, VeroE6 cells $\left(3.25 \times 10^{5} \mathrm{cell} / \mathrm{mL}\right)$ were seeded in 24 -well plates and incubated overnight. Prior to PRNT, patient sera were heat-inactivated at $56^{\circ} \mathrm{C}$ for $30 \mathrm{~min}$. For each dilution step (duplicate), patient sera were diluted in $220 \mu \mathrm{L}$ OptiPro and mixed 1:1 with 220 $\mu \mathrm{L}$ virus solution containing 100 plaque forming units. The $440 \mu \mathrm{L}$ serum-virus solution was gently vortexed and incubated at $37^{\circ} \mathrm{C}$ for $1 \mathrm{~h}$. Each 24 -well was incubated with $200 \mu \mathrm{L}$ serum-virus solution. After $1 \mathrm{~h}$ at $37^{\circ} \mathrm{C}$ supernatants were discarded, and cells were supplemented with $1.2 \%$ Avicel solution in DMEM. After 3 days at $37^{\circ} \mathrm{C}$, supernatants were removed and the 24 -well plates were fixed and inactivated using a $6 \%$ formaldehyde/PBS solution and stained with crystal violet as described. Serum dilutions with a plaque reduction of 50\% (PRNT50) and 90\% (PRNT90) are referred to as titers. Unless stated otherwise, cut-off titers were set at $<1: 20$. For numerical calculations, titers $>1: 640$ were set to 1:1,280. All plaque reduction neutralisation tests were performed in a central laboratory.

Enzyme-Linked Immunosorbent Assay (Euroimmun)

The Euroimmun anti-SARS-CoV-2 assay is a classical enzymelinked immunosorbent assay (ELISA) for the detection of IgG to the S1 domain of the SARS-COV-2 spike (S) protein and IgA to the $\mathrm{S} 1$ domain of the SARS-CoV-2 spike protein. The assay was performed manually according to the manufacturer's instructions as previously described [16]. Results are expressed as optical density (OD) ratios which were calculated based on the sample and calibrator OD values. For all analytes, a ratio $<0.8$ was considered to be non-reactive or negative. An OD-ratio of $\geq 1.1$ was considered to be positive for all analytes. All ELISA tests were performed in a central laboratory.

\section{Severity and Symptoms of the SARS-CoV-2 Infection of CCP}

Donors

For assessment of severity of the SARS-CoV-2 infection of the donors, a history of the absence/presence of the following 10 symptoms was taken: fever, chills, headache and/or myalgia, loss of taste and/or smell, rhinitis, sore throat, cough and/or chest pain, loss of appetite and/or nausea, diarrhoea, fatigue. The severity of COVID-19 in donors was classified as asymptomatic (no symptoms), mild (SARS-CoV-2 affects mainly the upper respiratory tract; key symptom is fever with or without cough), moderate (bronchopulmonary inflammation with key symptoms breathlessness, tachycardia, fever and cough), severe (at least one of the following: respiratory rate $\geq 30$ breaths/minute under ambient air; requirement of any type of ventilation support; needs intensive care).

\section{Statistical Analysis}

Summarized data are generally expressed as means \pm SD or medians and interquartile (IQR) and overall ranges (minimum maximum). Statistical differences between 2 data sets were assessed using Mann-Whitney test for unpaired samples. Differences between more than 2 data sets were calculated using a KruskalWallis test with correction for multiple comparisons by Dunn's test. Linear regression between 2 parameters was quantified by goodness of fit with $r^{2}$. Correlations between 2 data sets were calculated using Pearson's correlation coefficient and the $t$ distribution with $n$ - 2 degrees of freedom. $p$ values $<0.05$ were considered to indicate statistical significance, $p$ values $<0.005$ were considered to indicate highly statistical significance.

\section{Results}

\section{Donors}

We report here a cohort of 144 donors who had recovered from COVID-19 and donated convalescent plasma for the CAPSID trial (EudraCT 2020-001310-38). Median age of the donors was 40 years (IQR 31-52; range 2061 ), $41 \%$ were females and 59\% males. Female donors were younger than male donors $(p=0.051)$. Further demographic and clinical characteristics at baseline are presented in Table 1.

The vast majority of donors (97.9\%) had a mild or moderate course of COVID-19 (Fig. 1a). Only one donor had been hospitalized due to COVID-19. None of the donors had severe COVID-19. We counted the number of the following 10 symptoms per donor: fever, chills, headache and/or myalgia, loss of taste and/or loss of smell, rhinitis, sore throat, cough and/or chest pain, loss of appetite and/or nausea, diarrhoea and fatigue. The most frequent symptoms were loss of taste/loss of smell, headache/myalgia and cough (Table 1 ). The mean number of 
Table 1. Demographic and clinical characteristics of 144 CCP donors

\begin{tabular}{|c|c|}
\hline \multicolumn{2}{|l|}{ Gender, $n(\%)$} \\
\hline Female & $59(41)$ \\
\hline Male & $85(59)$ \\
\hline \multicolumn{2}{|l|}{ Age, years } \\
\hline Median & $p=0.051$ \\
\hline All & 40 \\
\hline Female & 37 \\
\hline Male & 42 \\
\hline \multicolumn{2}{|l|}{ IQR } \\
\hline All & $31.0-52.0$ \\
\hline Female & $28.0-51.0$ \\
\hline Male & $34.0-53.5$ \\
\hline \multicolumn{2}{|l|}{$\operatorname{Min} / \max$} \\
\hline All & $20-61$ \\
\hline Female & $20-61$ \\
\hline Male & $20-61$ \\
\hline \multicolumn{2}{|l|}{ Weight, kg } \\
\hline Median & $p<0.001$ \\
\hline All & 82 \\
\hline Female & 72 \\
\hline Male & 84 \\
\hline \multicolumn{2}{|l|}{ IQR } \\
\hline All & $71.3-93.0$ \\
\hline Female & $64.0-85.0$ \\
\hline Male & $78.0-95.0$ \\
\hline \multicolumn{2}{|l|}{ Blood group, $n(\%)$} \\
\hline 0 & $55(38.2)$ \\
\hline A & $62(43.1)$ \\
\hline B & $15(10.4)$ \\
\hline $\mathrm{AB}$ & $12(8.2)$ \\
\hline \multicolumn{2}{|c|}{ Donors with the following symptoms, $n=*$} \\
\hline Fever & 58 \\
\hline Chills & 16 \\
\hline Headache and/or myalgia & 67 \\
\hline Loss of taste/loss of smell & 72 \\
\hline Rhinitis & 26 \\
\hline Sore throat & 28 \\
\hline Cough and/or chest pain & 66 \\
\hline Loss of appetite and/or nausea & 6 \\
\hline Diarrhoea & 15 \\
\hline Fatigue & 47 \\
\hline
\end{tabular}

* The majority of donors had several symptoms (see Fig. 1).

symptoms per donor was 3.5 (Fig. 1b), and in the median, donors had 4 symptoms (IQR 3-4; range 0-9). Female donors reported a mean number of 3.7 symptoms and male donors a mean number of 3.3 symptoms $(p=0.19$; Fig. 1b). No symptoms were reported by $3.2 \%$ of the donors. Only $10.4 \%$ of the donors had experienced 6 or more symptoms. The number of COVID-19 symptoms reported by the CCP donors showed a weak linear correlation with the PRNT50 titers $\left(r^{2}=0.044 ; p=0.02\right)$.

\section{Donations}

Sixty-two donors (43\%) donated once, 35 donors (24\%) twice and 47 donors (33\%) donated 3 up to 8 times.
In total, the 144 donors underwent 319 apheresis procedures, that is, 2.2 plasmapheresis sessions per donor (online suppl. Fig. 1; for all online suppl. material, see www. karger.com/doi/10.1159/000515610). The median interval between diagnosis of the SARS-CoV-2 infection and the plasmapheresis procedures was 97.5 days (IQR 74122; range 33-249 days) for the first plasmapheresis and 116 days (IQR 90-139 days) with a range of 33 up to 251 for all plasmapheresis sessions.

The target volume of plasmapheresis was adapted to the body weight (see the Methods section). The body weight of female donors was significantly lower compared to male donors (Table 1). The median collection volume per plasmapheresis was $850 \mathrm{~mL}$. It was significantly higher in male donors $(850 \mathrm{~mL})$ than in female donors $(750 \mathrm{~mL} ; p<0.001)$. The volume was split into therapeutic units with a target volume of $250-325 \mathrm{~mL}$. We obtained a mean number of 2.7 units per plasmapheresis session (2.9 units/plasmapheresis from male and 2.6 units/plasmapheresis session from female donors; $p<$ $0.001)$. The median volume of the processed units was $281 \mathrm{~mL}$ (IQR 256-285; range 77-851 mL). Not all of these units were released and certified for use within the clinical trial.

\section{Antibody Titers}

Anti-SARS-CoV-2 IgG and IgA antibodies were measured by an ELISA and neutralizing antibody titers were measured by a PRNT test as described in Donors and Methods. Results were expressed as titer at which plaque formation was inhibited by 50 and $90 \%$. The distribution of PRNT50 and PRNT90 values of all plasmapheresis sessions are shown in Figure 2a, b, respectively. The coefficient of a linear correlation between PRNT50 and PRNT90 was $r^{2}=0.75(p<0.001)$.

We also analysed the PRNT50 titers in the subgroup of the 82 donors who donated at least twice. The titer of neutralizing antibodies in repeat donors showed different patterns: Figure 3 presents the PRNT50 titer of the last plasmapheresis of repeat donors as relative ratio compared to the PRNT50 titer of the first plasmapheresis. A ratio of 1 means that the PRNT50 titer of the repeat plasmapheresis sessions remained stable, ratios $>1$ indicate an increase and ratios $<1$ indicate a decrease of PRNT50 titers compared to first plasmapheresis. As shown in Figure 3, we observed stable, decreasing or increasing titers measured at repeat plasmapheresis sessions (Fig. 3a). We compared the PRNT50 titer in the first donation and the last donation of these donors irrespective of the total number of donations. The mean PRNT50 titer at time of the first donation was 1:86 compared to 1:87 at the last donation ( $p=0.3$; Fig. $3 \mathrm{~b}$ ). The median of the first donation was 1:80 (IQR 1:20-1:160; range $0-1: 320$ ) and 1:40 (IQR 1:20-1:80; range 0-1:640) for the second donation. 


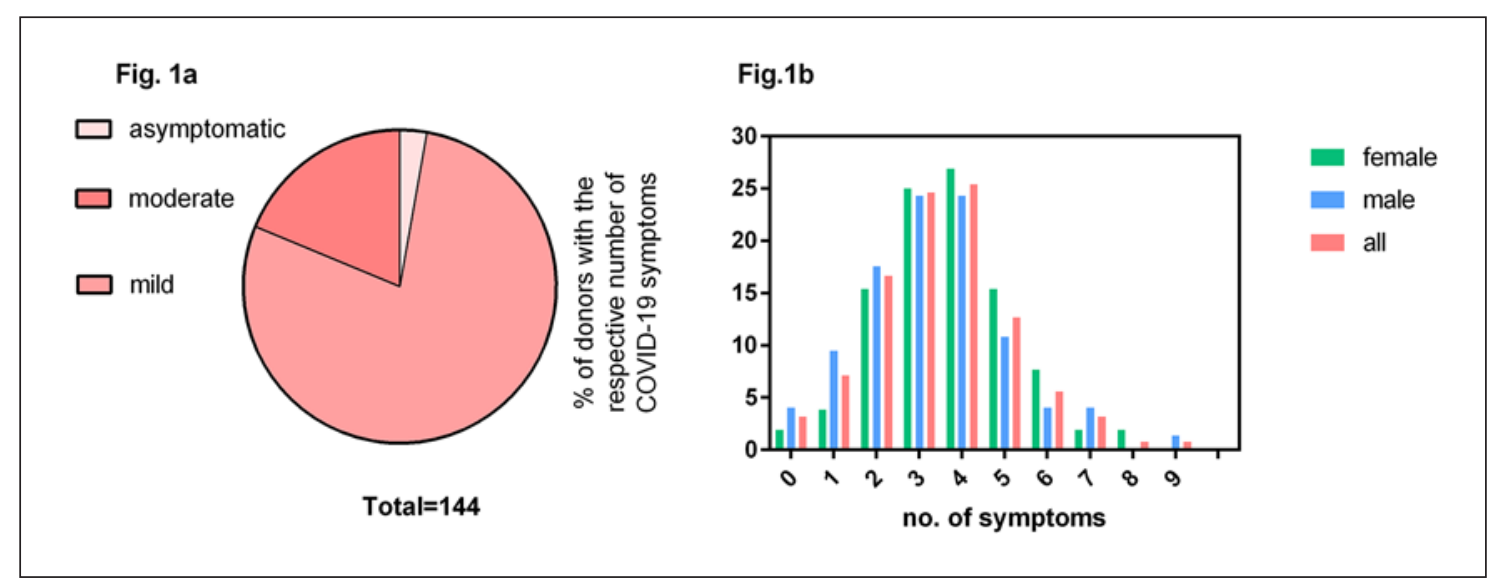

Fig. 1. COVID-19 disease in CCP donors. a Severity of disease in the CCP donor population $(n=144)$. b Proportion with the respective number of the following 10 COVID-19 symptoms: fever, chills, headache and/or myalgia, loss of taste and/or smell, rhinitis, sore throat, cough and/or chest pain, loss of appetite and/or nausea, diarrhoea, fatigue. This analysis of number of symptoms refers to 126 donors; in the other 18 donors no sufficient data were available.

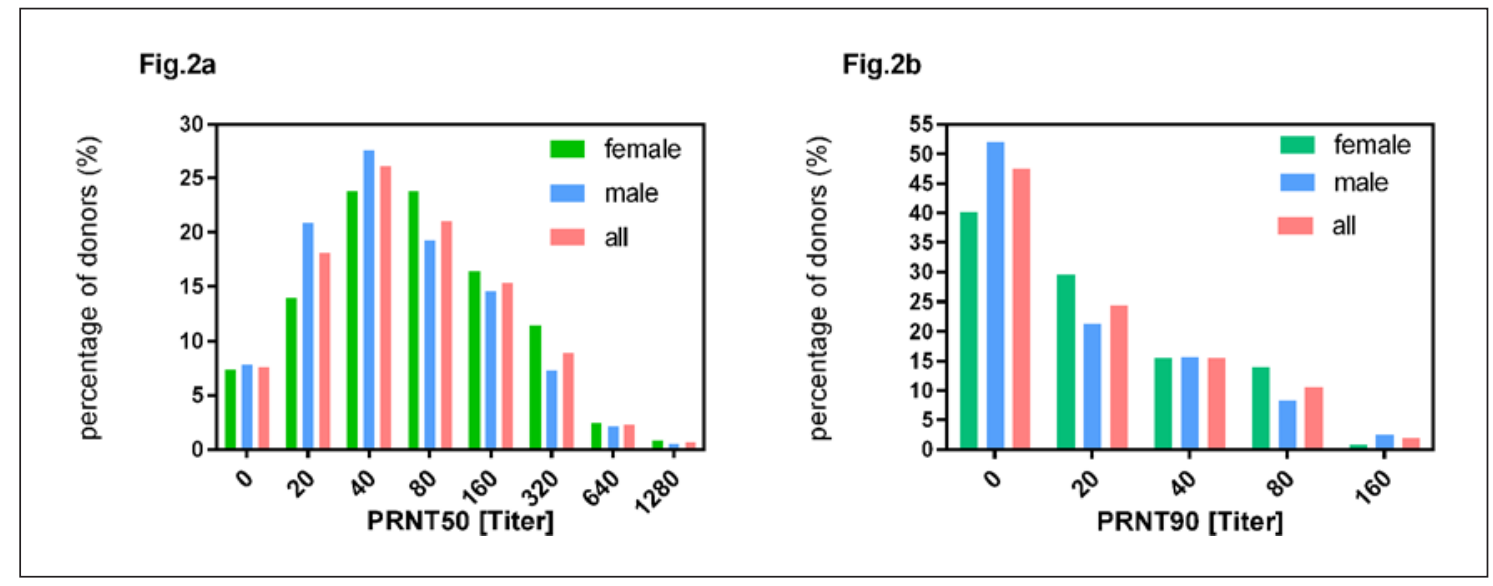

Fig. 2. Distribution of neutralizing antibody titers expressed as PRNT50 (a) and PRNT90 (b) for plasmapheresis sessions of female and male donors and all donors $(n=314)$.

Figure $3 \mathrm{c}$ shows that increasing PRNT50 titers could be observed after a longer interval from diagnosis. Titers of 1:160 or higher could be detected at the earliest 49 days after diagnosis. These higher titers could be seen until day 251. The mean PRNT50 titers remained stable over consecutive plasmapheresis procedures (Fig. $3 \mathrm{~d}$ ).

For the correlation of titer levels with demographic characteristics of donors, we used the highest titer of each donor measured on occasion of consecutive plasmapheresis procedures.

PRNT50 titers at first plasmapheresis and also highest PRNT50 titers did not correlate with age of the donors (coefficient of linear regression between donor and highest PRNT50 titer $r^{2}=0.014 ; p=0.17$; Fig. $4 \mathrm{a}$ ). The mean of the highest PRNT50 titer of each donor was 136 in females and 122 in males ( $p=0.30$; Fig. $4 \mathrm{~b}$ ). The mean of the highest PRNT50 titers of each donor were 141 in donors with blood group 0, 129 in group A donors, 96 in group $\mathrm{B}$ donors and 98 in group $\mathrm{AB}$ donors (no significant difference; Fig. 4c). The mean of the highest PRNT50 titers of donors with $0-3$ and $\geq 4$ reported COVID-19 symptoms was 99 and 142 , respectively ( $p=0.023$; Fig. $4 d$ ).

The ratios of anti-SARS-CoV-2 IgA and IgG antibodies in the ELISA versus the interval between diagnosis of the SARS-CoV-2 infection and the plasmapheresis sessions are shown in Figure 5a, b. The coefficients of correlation $r^{2}$ between interval since diagnosis and antiSARS-CoV-2 IgA or IgG ratio were 0.015 and 0.014 ( $p=$ $0.050, p=0.053$ ). The correlation between the IgA and IgG ratios measured by ELISA and the PRNT50 titers are shown in online supplementary Figure 2. The coefficient of correlation between IgA ratios and PRNT50 titers was 
Fig. 3a

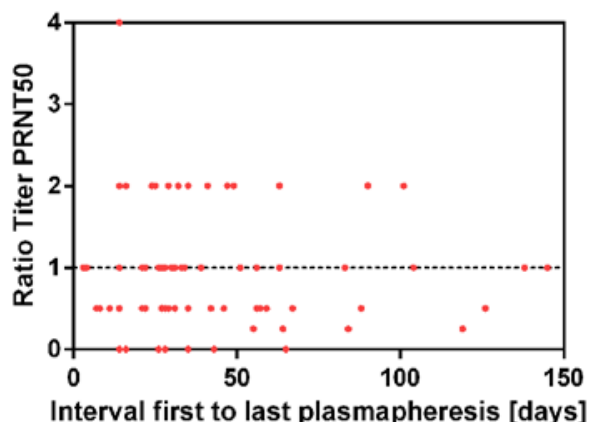

Fig. 3c

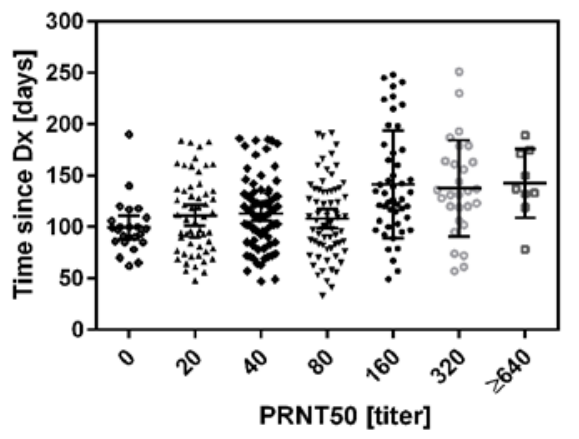

Fig.3b

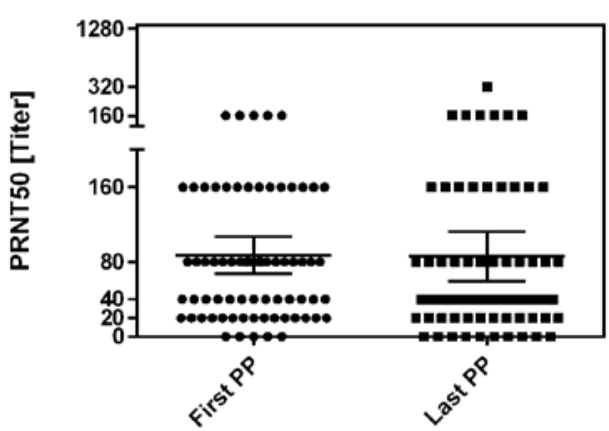

Fig.3d

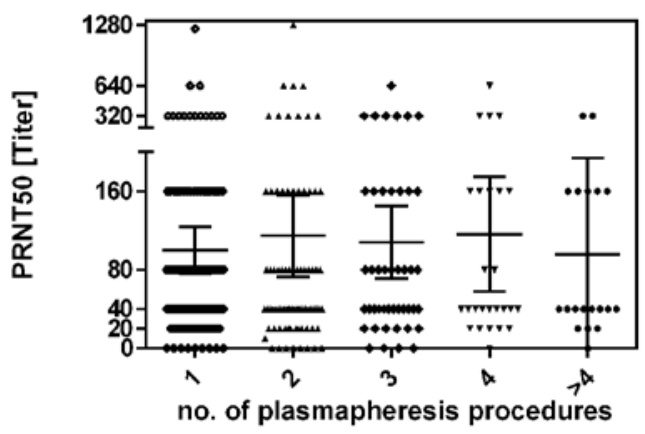

Fig. 3. Development of PRNT50 titers over time. PRNT50 of first and last plasmapheresis sessions were analysed. The median interval was 33.5 days (range 3-145 days). a Ratios of PRNT50 of first and last plasmapheresis sessions were plotted against the interval. Values below 1 indicate a decline and values of 1 or higher indicate stable or increasing PRNT50 titers in the observational period. b PRNT50 titers at the first and last plasmapheresis session of repeat donors (irrespective of total number of donations). The hori- zontal lines and error bars in (b) indicate mean titers $\pm 95 \%$ CIs. First plasmapheresis occurred at day 92.5 (median, range 33-230) and last plasmapheresis at day 135.0 (median, range 63-251). c Higher PRNT50 titers could be observed after longer intervals from diagnosis. $\mathbf{d}$ PRNT50 titers measured at first and subsequent plasmapheresis sessions. The horizontal lines and error bars in (b) indicate mean titers $\pm 95 \%$ CIs. PP, plasmapheresis. $0.18(p<0.0001)$ and between IgG ratios and PRNT50 it was $0.30(p<0.0001)$. Selection of CCP with an antiSARS-CoV-2 IgA ratio above the 25th percentile (ratio > 1.298) or an anti-SARS-CoV-2 IgG ratio above the 25th percentile (ratio > 2.195) enriched for CCP units with higher PRNT50 titers, in particular when both IgG and IgA ratios were above the 25th percentile (Fig. 5c; $p<$ 0.001 for CCP donations with a ratio $>25$ th percentile for IgG alone or IgG + IgA compared to donations with both IgG and IgA ratio in the lower quartile).

\section{Discussion}

Here, we report a series of 319 plasmapheresis collections from 144 donors. The antibody levels varied greatly between CCP donors. Similar heterogeneity of humoral antibody response in CCP donors has been reported by others [19-25]. About 27\% of CCP donations in our series had a
PRNT50 titer of 160 or higher. Therefore, we searched for predictive markers to select donors with high-titer neutralizing antibodies. We could not find a correlation of neutralizing antibody titers with gender, age or $\mathrm{ABO}$ blood type. There was a positive correlation between higher PRNT50 titers and a higher number of COVID-19 symptoms reported by the donor and a longer interval between SARS-CoV-2 diagnosis and plasmapheresis.

Our observations are in line with other reports who could not find a significant correlation of titers of neutralizing antibodies with gender in univariate analysis [19, 20, 24]. Some studies have reported significant correlation of antibody titers with increasing age, yet with little predictive value [19-21, 26]. In our cohort, this correlation between neutralising antibody titer and age is not significant. A stronger association of antibody levels with age was observed in an Italian study which included a subgroup of hospitalized patients, some of which had even required mechanical ventilation [22]. Additionally, the interval between 


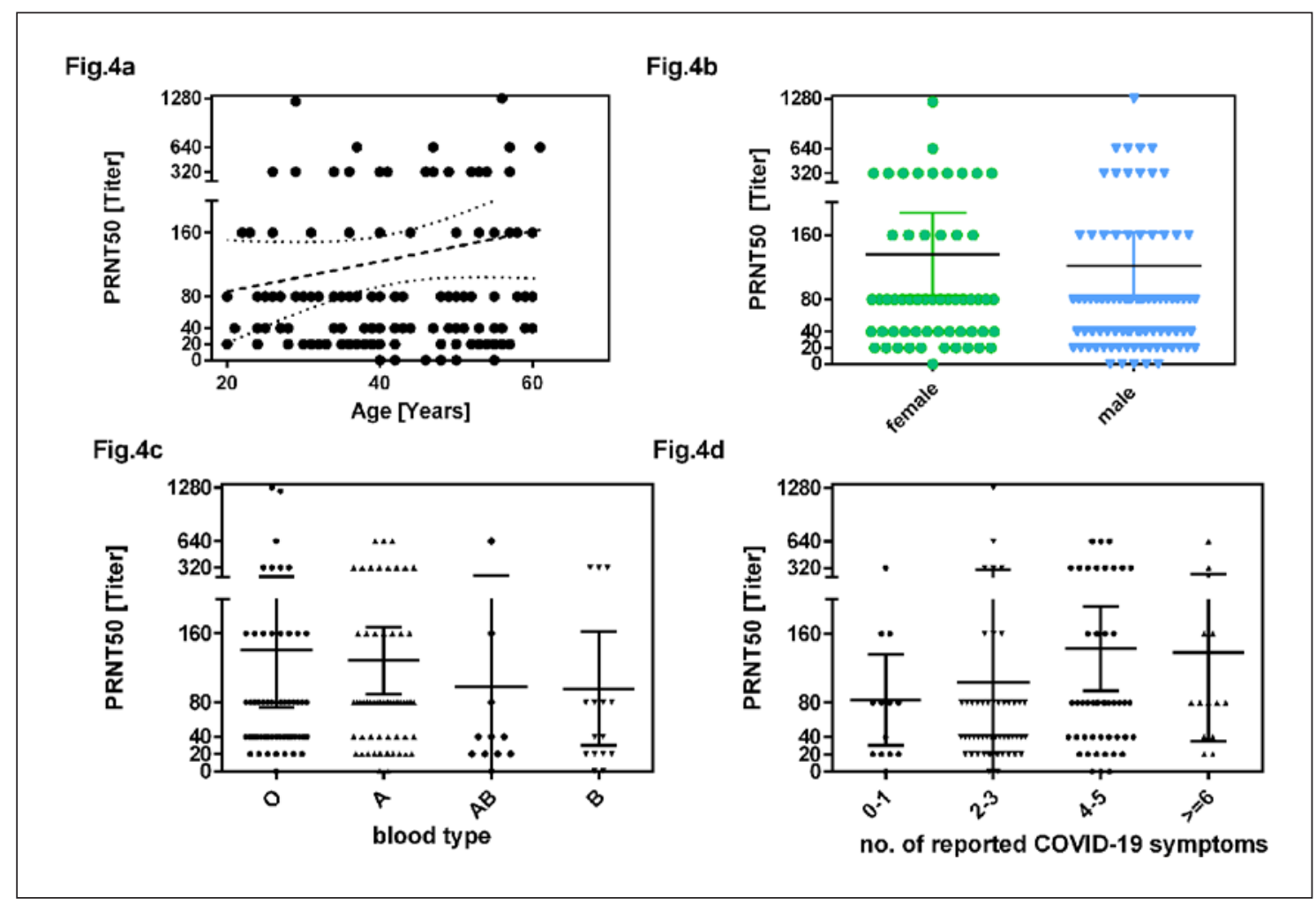

Fig. 4. Linear regression between age and neutralizing antibody titers expressed as PRNT50 (a). The dashed line shows the linear regression with $95 \%$ confidence limit (dotted line). b PRNT50 titer by gender. c PRNT50 titer by ABO blood type. d PRNT50 titer by number of reported COVID-19 symptoms (0-1 symptoms, 2-3,
4-5 and $\geq 6$ symptoms [fever, chills, headache and/or myalgia, loss of taste and/or loss of smell, rhinitis, sore throat, cough and/or chest pain, loss of appetite and/or nausea, diarrhoea and fatigue]). The horizontal lines and error bars in (b-d) indicate mean titers \pm 95\% CIs. PRNT50 titers are peak titers of each donor. resolution of symptoms and plasmapheresis was rather short in this cohort [22]. Other studies reported a correlation between body weight and neutralizing antibody titers $[24,25]$. We could not confirm this in our cohort.

It has been reported that titers of neutralizing antibodies are associated with disease severity $[19,22,26,27]$. We cannot analyse this in our CCP cohort since none of the donors had severe COVID-19. It is in line with other reports that CCP donors predominantly represent patients who had mild or moderate disease without hospitalization [19-21]. However, if the patients with mild or moderate COVID-19 were divided in subgroups according to the number of reported COVID-19 symptoms, we observed a correlation between number of symptoms and PRNT50 titers.

The accrual of CCP donors in our cohort was mainly based on press releases, media reports and a webpage providing information for convalescent individuals. This approach seems to primarily motivate donors who had suffered from typical COVID-19 symptoms but had not experienced severe complications.

While the gold standard for assessment of the neutralizing potency of CCP are PRNT assays or microneutral- ization assays, they are time-consuming and require biosafety level 3 laboratories. Immunoassays for anti-SARSCoV-2 antibodies are an option for rapid and high-throughput measurement of the antibody content of CCP $[16,28,29]$. We observed a positive but weak correlation between the IgG and IgA ratios measured by an ELISA and the PRNT50 titers. The selection of CCP donations with higher ratios of IgG and IgA in the ELISA helps to enrich for CCP with higher levels of neutralization $[16,20,21]$. The exclusion of donations with ratios of IgG and IgA in the lower quartile in the ELISA selects for CCP with higher neutralizing PRNT50 titers.

Several studies reported a decline in neutralizing antibody titers over time, that is, in longitudinal follow-up of repeat plasma collections $[19,23-25,30,31]$. In our series, the comparison of neutralizing antibody titers (measured by a PRNT assay) of consecutive donations and the comparison of the neutralizing antibody titers in the first and last donation of all donors with at least 2 donations demonstrate a stable antibody level. We could confirm this finding on a subset of CCP donors by a neutralisation test based on the inhibition of SARS-CoV-2 receptor binding domain interaction with the angiotensin-converting enzyme 2 pro- 


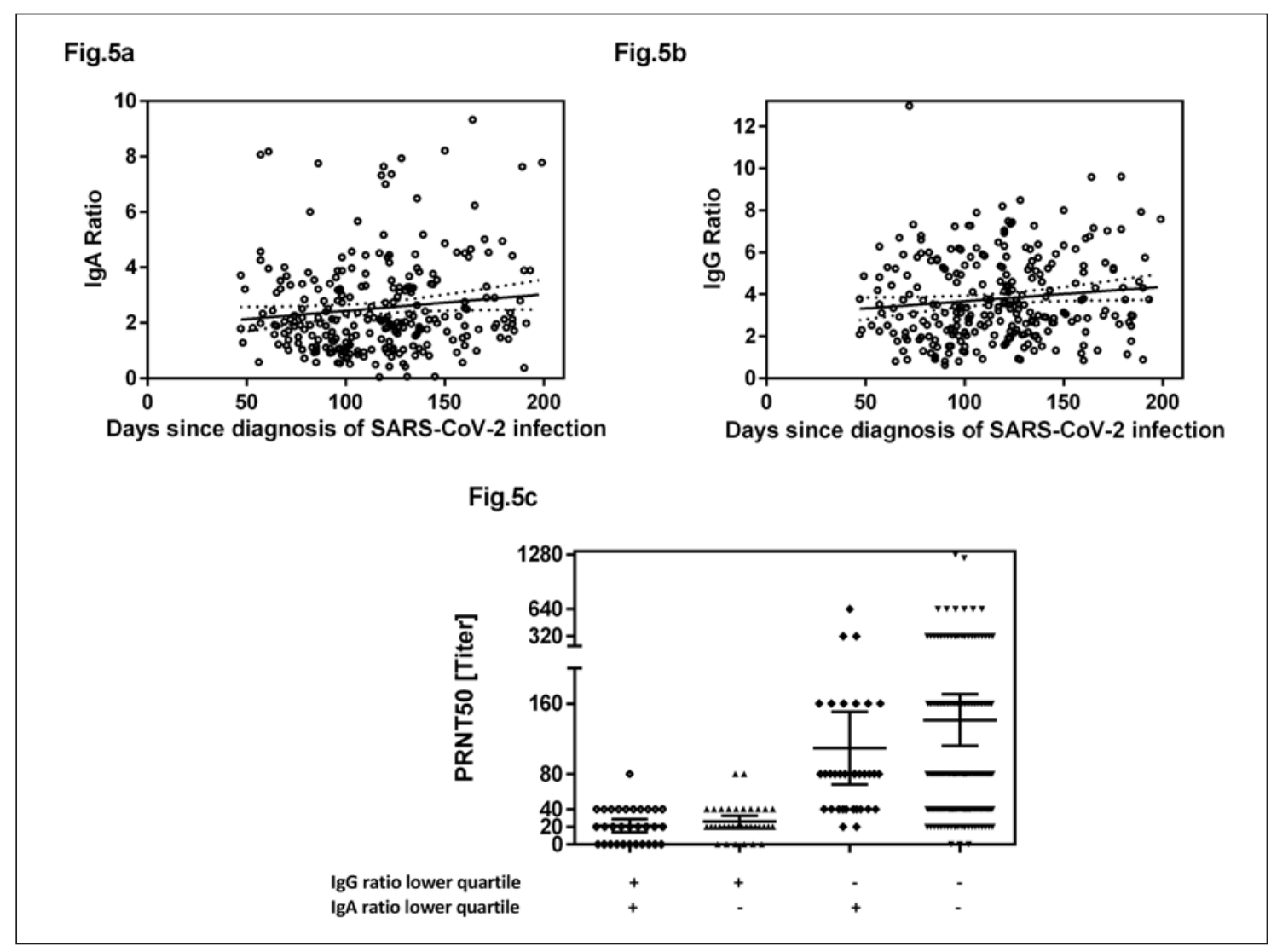

Fig. 5. Linear regression of the interval from diagnosis of SARS$\mathrm{CoV}-2$ infection in CCP donors to plasmapheresis procedures and IgA antibodies (a) and IgG antibodies (b) measured by an ELISA. The lines show the linear regression and the dotted lines the 95\% confidence limits. c PRNT50 titer grouped by anti-SARS-CoV-2 IgG and IgA ratios in the Euroimmune ELISA. The first column presents the PRNT50 titer of CCP which were in the lower quartile for both IgG and IgA. The second and third columns represent the titers of CCP which were in the lower quartile for either IgG or IgA and the fourth column for CCP with neither IgG nor IgA ratio in the lower quartile of the Euroimmune ELISA. The horizontal lines and error bars in (c) indicate mean titers $\pm 95 \%$ CIs. tein [32]. Thus, there is no evidence that repeat plasmapheresis procedures cause a decline of antibody titers. Furthermore, a proportion of our donors reached the peak PRNT50 titers during follow-up after the first plasmapheresis. Another study which reported waning of SARS-CoV-2 RBD antibodies in longitudinal CCP samples used $>84$ days from onset of symptoms to diagnosis as a cut-off for "late" CCP donations. If we compare the neutralizing antibody titers between the first plasmapheresis and repeat plasmaphereses $>84$ days after diagnosis of the SARS-CoV-2 infection, we cannot find a significant decline.

We did not invite donors with low and/or substantially declining neutralization titers for subsequent plasmapheresis procedure. Thus, the observed time course does not merely reflect the natural course of humoral immunity but is influenced by donor selection. On the other hand, this demonstrates that it is possible to serially collect CCPs with a rather stable neutralizing capacity by appropriate selection of donors.

In summary, our results and other published series on CCP donor cohorts demonstrate substantial heterogene- ity of titers of neutralizing antibodies. Some studies found correlation of neutralizing antibody titers with donor factors. However, some of these findings could not be confirmed in other reports including ours. Moreover, the predictive value of these correlations is low. Therefore programmes for CCP donor accrual should not upfront exclusively enrol donors of specific gender, age group, severity of COVID-19 or time since COVID-19. Based on our experience, we propose to implement a broad screening approach. By selecting donors with stable or increasing antibody levels for repeat donations, one can establish a donor population providing CCPs with stable antibody level even several months after the SARS-CoV-2 infection of the donors. There is evidence for better therapeutic effects of high-titer CCPs, however, mainly from non-randomized trials [2]. This needs to be confirmed by randomized clinical trials. Standardisation of the antibody tests used is urgently needed. It should be taken into account that mechanisms of action other than passive transfer of (neutralizing) antibodies can contribute to the therapeutic efficacy of CCP [33]. 


\section{Acknowledgements}

We thank all CCP donors for their ready availability to contribute to this programme. We acknowledge all those involved in the process from collection to testing, manufacturing and supply into the hospital and the staff of the CRO Alcedis $\mathrm{GmbH}$. In addition, we acknowledge the following local study team members: Marianne Holl, Dzenan Kilalic, Ramin Lotfi (Ulm), Eva Mayer (Homburg), Jörg Martens (Hannover), Veronika Brixner, Kai Hourfar, Stefanie Herkt, Patricia Manns, Knut Werner (Frankfurt) and Stefanie Nowak-Harnau (Tübingen).

\section{Statement of Ethics}

The study complies with the guidelines for human studies and was conducted ethically in accordance with the World Medical Association Declaration of Helsinki. The CAPSID trial "A randomized, prospective, open label clinical trial on the use of convalescent plasma compared to best supportive care in patients with severe COVID-19"(CAPSID;Eudra-CT2020-001310-38,NCT04433910) has been approved by the competent regulatory authorities in Germany (Paul-Ehrlich-Institut) and by the Ethical Committee of the University of Ulm and the Ethical Committees of participating centres. All donors gave their informed consent for donation and participation in the research project of the clinical trial.

\section{Conflict of Interest Statement}

Dr. Victor M. Corman is named together with Euroimmun on a patent application filed recently regarding the diagnostic of SARS-CoV-2 by antibody testing. All other authors have no conflicts of interest to declare.

\section{Funding Sources}

The randomized clinical trial CAPSID including the preparation of convalescent plasma units is supported by the Bundesministerium für Gesundheit (“German Federal Ministry of Health”).

\section{Gefördert durch:}

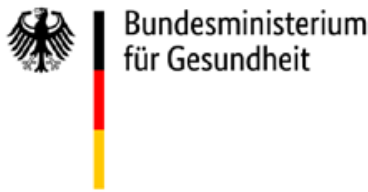

\section{aufgrund eines Beschlusses des Deutschen Bundestages}

\section{Author Contributions}

H.S. and S.K. designed the clinical trial, wrote the clinical trial protocol and served as principle investigator and trial coordinator; H.S., S.K., B.J., and V.M.C. analysed data; H.S., S.K., B.J., V.M.C., T.S., and C.D. analysed SARS-CoV-2 antibodies; J.P., P.W., R.B., R.M., T.T., T.B., R.S., D.J., N.G., T.B., H.E., and H.K. cared for plasma donors and collected data; M.S. performed infectious disease marker testing; T.A. managed transport of laboratory samples and collected data; H.S., S.K., T.A., and E.S. designed the research project. H.S. and S.K. wrote the manuscript.

\section{References}

1 Chai KL, Valk SJ, Piechotta V, Kimber C, Monsef I, Doree C, et al. Convalescent plasma or hyperimmune immunoglobulin for people with COVID-19: a living systematic review. Cochrane Database Syst Rev. 2020 Oct; 10:CD013600.

2 Joyner MJ, Senefeld JW, Klassen SA, Mills JR, Johnson PW, Theel ES, et al. Effect of Convalescent Plasma on Mortality among Hospitalized Patients with COVID-19: Initial ThreeMonth Experience. medRxiv. 2020, Preprint.

3 Liu ST, Lin HM, Baine I, Wajnberg A, Gumprecht JP, Rahman F, et al. Convalescent plasma treatment of severe COVID-19: a propensity score-matched control study. Nat Med. 2020 Nov;26(11):1708-13.

4 Li L, Zhang W, Hu Y, Tong X, Zheng S, Yang J, et al. Effect of Convalescent Plasma Therapy on Time to Clinical Improvement in Patients With Severe and Life-threatening COVID-19: A Randomized Clinical Trial. JAMA. 2020 Aug;324(5):460-70.

5 Gharbharan A, Jordans CCE, GeurtsvanKessel C, den Hollander JG, Karim F, Mollema FPN, et al. Convalescent Plasma for COVID-19. A randomized clinical trial. medRxiv. 2020, Preprint.
6 Avendano-Sola C, Ramos-Martinez A, Munez-Rubio E, Ruiz-Antoran B, Malo de Molina R, Torres F, et al. Convalescent Plasma for COVID-19: A multicenter, randomized clinical trial. medRxiv 2020, Preprint.

7 Agarwal A, Mukherjee A, Kumar G, Chatterjee P, Bhatnagar T, Malhotra P; PLACID Trial Collaborators. Convalescent plasma in the management of moderate covid-19 in adults in India: open label phase II multicentre randomised controlled trial (PLACID Trial). BMJ. 2020 Oct;371:m3939.

8 Simonovich VA, Burgos Pratx LD, Scibona P, Beruto MV, Vallone MG, Vazquez C, et al. A Randomized Trial of Convalescent Plasma in Covid-19 Severe Pneumonia. N Engl J Med. 2021 Feb;384(7):619-29.

9 Libster R, Perez MG, Wappner D, Coviello S, Bianchi A, Braem V, et al. Early High-Titer Plasma Therapy to Prevent Severe Covid-19 in Older Adults. N Engl J Med. 2021 Feb 18; 384(7):610-8

10 Libster R, Marc GPr, Wappner D, Coviello S, Bianchi A, Braem V, et al. Prevention of severe COVID-19 in the elderly by early hightiter plasma. medRxiv 2020, Preprint.
11 Katz LM. (A Little) Clarity on Convalescent Plasma for Covid-19. N Engl J Med. 2021 Feb; 384(7):666-8.

12 Körper S, Jahrsdorfer B, Appl T, Klüter H, Seifried E, Schrezenemeier H. Convalescent plasma for treatment of severe COVID-19: rationale and designing of a randomized, openlabel clinical trial of convalescent plasma compared to best supportive care (CAPSID Trial). Transfusionsmedizin. 2020;10:13542.

13 Vorstand der Bundesärztekammer auf Empfehlung seines Wissenschaftlichen Beirats und im Einvernehmen mit dem PaulEhrlich-Institut: Richtlinie zur Gewinnung von Blut und Blutbestandteilen und zur Anwendung von Blutprodukten (Richtlinie Hämotherapie); Köln, Deutscher Ärzteverlag, 2017.

14 Müller MM, Fraile MI, Hourfar MK, Peris LB, Sireis W, Rubin MG, et al. Evaluation of two, commercial, multi-dye, nucleic acid amplification technology tests, for $\mathrm{HBV} / \mathrm{HCV} / \mathrm{HIV}$ 1/HIV-2 and B19V/HAV, for screening blood and plasma for further manufacture. Vox Sang. 2013 Jan;104(1):19-29. 
15 Hourfar K, Eberle J, Müller M, Micha Nübling C, Chudy M, Kress J, et al. Human immunodeficiency virus 1 dual-target nucleic acid technology improves blood safety: 5 years of experience of the German Red Cross blood donor service Baden-Württemberg-Hessen. Transfusion. 2018 Dec;58(12):2886-93.

16 Jahrsdörfer B, Kroschel J, Ludwig C, Corman VM, Schwarz T, Körper S, et al. Independent side-by-side validation and comparison of four serological platforms for SARS-CoV-2 antibody testing. J Infect Dis. 2020 Oct;jiaa656.

17 Wölfel R, Corman VM, Guggemos W, Seilmaier M, Zange S, Müller MA. Virological assessment of hospitalized cases of coronavirus disease 2019. Nature. 2020;581:465-9.

18 Kreye J, Reincke SM, Kornau HC, SánchezSendin E, Corman VM, Liu H, et al. A Therapeutic Non-self-reactive SARS-CoV-2 Antibody Protects from Lung Pathology in a COVID-19 Hamster Model. Cell. 2020 Nov; 183(4):1058-69.e19.

19 Mehew J, Johnson R, Roberts D, Harvala H. Convalescent plasma for COVID-19: male gender, older age and hospitalisation associated with high neutralising antibody levels, England, 22 April to 12 May 2020. Euro Surveill. 2020 Nov;25(45):2001754-7917.

20 Jungbauer C, Weseslindtner L, Weidner L, Gansdorfer S, Farcet MR, Gschaider-Reichhart E, et al. Characterization of 100 sequential SARS-CoV-2 convalescent plasma donations. Transfusion. 2021 Jan;61(1):12-6.

21 Gniadek TJ, Thiede JM, Matchett WE, Gress AR, Pape KA, Fiege JK, Jenkins MK, Menachery VD, Langlois RA, Bold TD: SARS-CoV-2 neutralization and serology testing of COVID-19 convalescent plasma from donors with nonsevere disease. Transfusion. 2021 Jan;61(1):17-23.
22 Del FC, Franchini M, Baldanti F, Percivalle E, Glingani C, Marano G, Mengoli C, Mortellaro C, Viarengo G, Perotti C, Liumbruno GM: A retrospective study assessing the characteristics of COVID-19 convalescent plasma donors and donations. Transfusion. 2020, Online ahead of print.

23 Perreault J, Tremblay T, Fournier MJ, Drouin $\mathrm{M}$, Beaudoin-Bussières $\mathrm{G}$, Prévost $\mathrm{J}$, et al. Waning of SARS-CoV-2 RBD antibodies in longitudinal convalescent plasma samples within 4 months after symptom onset. Blood. 2020 Nov;136(22):2588-91.

24 Wendel S, Kutner JM, Machado R, FontãoWendel R, Bub C, Fachini R, et al. Screening for SARS-CoV-2 antibodies in convalescent plasma in Brazil: preliminary lessons from a voluntary convalescent donor program. Transfusion. 2020 Dec;60(12):2938-51.

25 Hähnel V, Peterhoff D, Bäuerlein V, Brosig AM, Pamler I, Johnson C, et al. Manufacturing of convalescent plasma of COVID-19 patients: aspects of quality. PLoS One. 2020 Dec; 15(12):e0243967.

26 Terpos E, Politou M, Sergentanis TN, Mentis A, Rosati M, Stellas D, et al. Anti-SARSCoV-2 Antibody Responses in Convalescent Plasma Donors Are Increased in Hospitalized Patients; Subanalyses of a Phase 2 Clinical Study. Microorganisms. 2020 Nov; 8(12): 1885.

27 Bošnjak B, Stein SC, Willenzon S, Cordes AK, Puppe W, Bernhardt G, et al. Low serum neutralizing anti-SARS-CoV-2 S antibody levels in mildly affected COVID-19 convalescent patients revealed by two different detection methods. Cell Mol Immunol. 2020 Nov.
28 Wouters E, Steenhuis M, Schrezenmeier H, Tiberghien P, Harvala H, Feys HB, et al. Evaluation of SARS-CoV-2 antibody titers and potency for convalescent plasma donation: a brief commentary. Vox Sang. 2020, Online ahead of print.

29 von Rhein C, Scholz T, Henss L, KronsteinWiedemann R, Schwarz T, Rodionov RN, et al.; von RC. Scholz T, Henss L, Kronstein-Wiedemann R, Schwarz T, Rodionov RN, Corman VM, Tonn T, Schnierle BS: comparison of potency assays to assess SARS-CoV-2 neutralizing antibody capacity in COVID-19 convalescent plasma. J Virol Methods. 2021; 288:114031.

30 Harvala H, Mehew J, Robb ML, Ijaz S, Dicks $\mathrm{S}$, Patel $\mathrm{M}$, et al. Convalescent plasma treatment for SARS-CoV-2 infection: analysis of the first 436 donors in England, 22 April to 12 May 2020. Euro Surveill. 2020 Jul;25(28): 2001260.

31 Beaudoin-Bussieres G, Laumaea A, Anand SP, Prevost J, Gasser R, Goyette G, et al. Decline of Humoral Responses against SARSCoV-2 Spike in Convalescent Individuals. mBio. 2020 Oct;11(5):e02590-20.

32 Jahrsdorfer B, Corman V, Korper S, Drosten C, Schrezenemeier H. Stable anti-SARSCoV-2 neutralisation titers in convalscent plasma donors over 7 months after symptom onset; 2021, submitted.

33 Askenase PW: COVID-19 therapy with mesenchymal stromal cells (MSC) and convalescent plasma must consider exosome involvement: Do the exosomes in convalescent plasma antagonize the weak immune antibodies? J Extracell Vesicles. 2020; 10:e12004. 